\title{
Statistical starting points in Africa
}

I $\mathrm{t}$ often seems as if health statistics in Africa are as scarce as oases in the desert. The wrong problem is identified or the wrong problem solved because the numbers are wrong or simply a mystery.

The situation was so dire that the World Health Organization felt compelled to produce a 330-page primer in 2003 on how developing nations should collect and use health statistics to determine areas of priority investment to achieve desired health outcomes.

The Guide to producing national health accounts with special applications for low-income and middle-income countries urged the adoption of a system measuring national health expenditures that was "designed to answer precise questions about a country's health system" (www.who.int/nha/docs/English _PG.pdf). "They provide a systematic compilation and display of health expenditure. They can trace how much is being spent, where it is being spent, what it is being spent on and for whom, how that has changed over time, and how that compares to spending in countries facing similar conditions. They are an essential part of assessing the success of a health system and of identifying opportunities for improvement. In the long term, a country can institutionalize the health accounts process and produce a time series of standardized tables, permitting a more thorough assessment of the progress being made toward national goals for the health system."

Has much changed over the course of the decade?

Perhaps, a little, in some countries.

Several countries have improved their health record keeping, using national health accounts, including Burkina Faso, Republic of Congo, Egypt, Kenya, Liberia, Namibia, Tanzania and Uganda.

But major holes, and challenges, remain.

"Some countries lack capacity to carry out tracking exercises and estimations; others produce information that

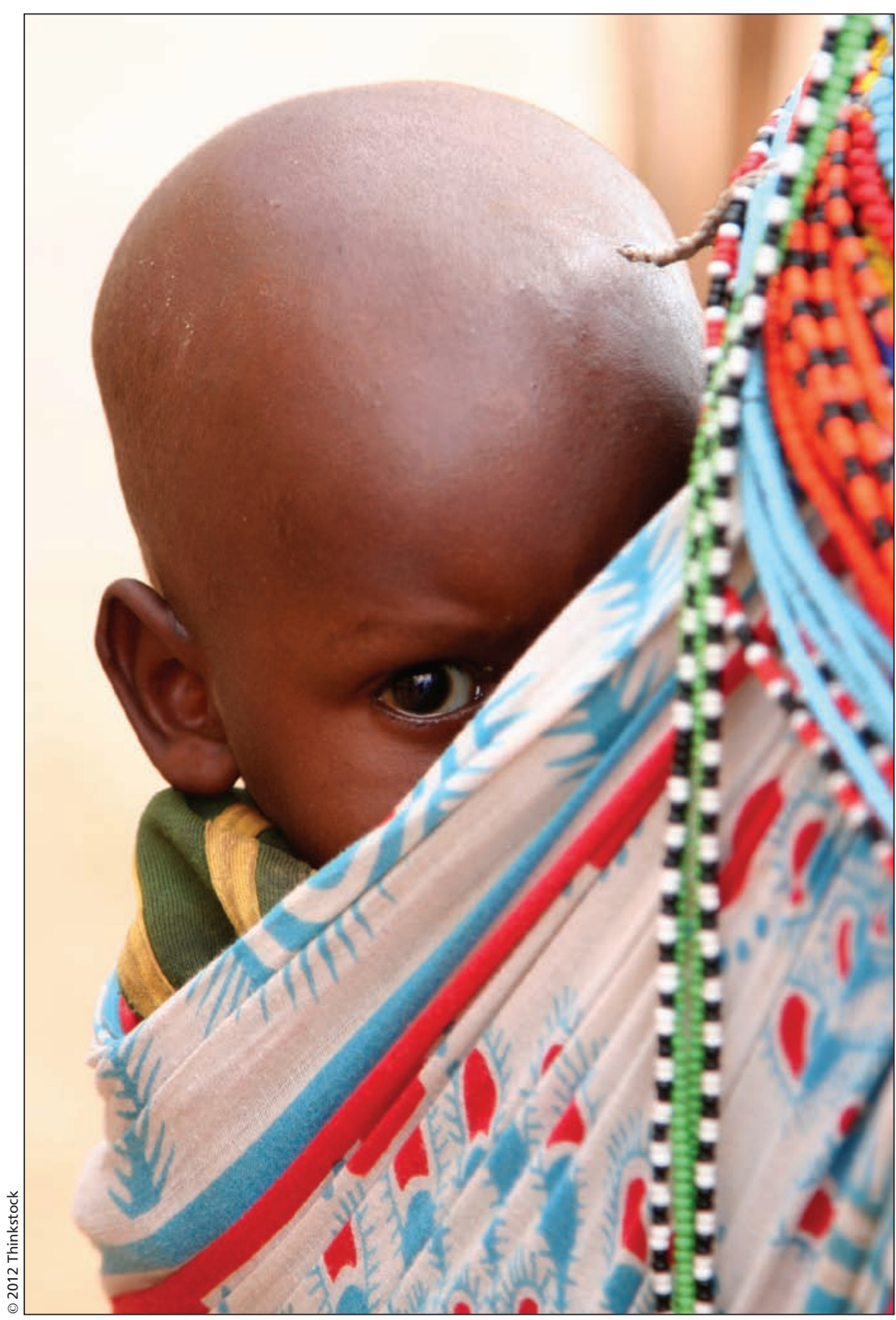

In some countries, the adoption of national health accounts have led to funding initiatives aimed at improvements in maternal and child health.

policymakers fail to use," according to a report by Health Systems 20/20, a six-year project that was funded by the United States Agency for International Development to promote the development of record keeping in Africa (www .healthsystems2020.org/files/2586_file _Resource_Tracking_f.pdf). "Many cannot afford the staff time and other costs of carrying out numerous and overlapping resource tracking methodologies: tracking often is done only 
with donor funding and external technical assistance, and the uncoordinated findings are less likely to be used for policy making."

Financing is a major obstacle, says Catherine Connor, deputy director of the project. The best way to get quality data is a household survey, which is quite expensive, she notes.

Another obstacle, Connor adds, is that most African countries have decentralized many government operations and thus do not have well-developed centralized information gathering and management systems to aggregate data collected at the district level, which makes national health resource tracking problematic.

Trust is also a major obstacle in the collection of health data, says André Zida, a health economist in Ouagadougou, Burkina Faso. "When we began collecting expenditure data from government and private sector organizations to prepare national health accounts, sometimes people would refuse to give us data because they thought we would be collecting more taxes from them."

Other obstacles include difficulty in obtaining and retaining staff capable of overseeing national account systems, he adds.

But there have been some successes, Zida says, citing the 2005 findings of a national health accounts (NHA) effort that led to redistribution of health funds after data indicated that the two poorest regions of Burkina Faso, with $20 \%$ of the country's population, got just $11 \%$ of health funding, while a wealthier region with $9 \%$ of the population tapped $29 \%$ of available health funds.

Countries that have adopted national health accounts have also been able to reassure international aid agencies that donated monies are actually spent on health programming, Zida says.

National health accounts also appear to have resulted in changes in health spending within several nations.

In Tanzania, a national health account effort revealed the need to diversify international donations across the spectrum of the health sector, says Mariam Ally, head of health care financing at the Ministry of Health and Social Welfare. "We also found out that most of the donor funding went into HIV programs, so we had to apply for more funding for health systems strengthening."
In Uganda, the 2006 national health account led to the allocation of US\$124 million to improve maternal and child health, says Candia Tom Aliti, principal finance officer with the Ministry of Health's Directorate of Planning and Development.

Similarly, use of accounts led to significant new health spending in Kenya, says Thomas Maina, national health accounts coordinator for the country. "The minister for health was able to obtain about $30 \%$ increase in budgetary support for the health sector using the NHA findings as an advocacy tool."

Given those indicators of the value of national health accounts, efforts are being made to expand them in some nations and to train more experts on the collection of health data. To that end, Uganda is seeking to incorporate national account health questions in its national household survey, while in Kenya, an attempt is being made to introduce a master's program in health economics at the University of Nairobi. Bernard Appiah, College Station, Tex.

CMAJ 2012. DOI:10.1503/cmaj.109-4182 